\title{
Long noncoding RNA SNHGI5 serves as an oncogene and predicts poor prognosis in epithelial ovarian cancer
}

This article was published in the following Dove Press journal:

OncoTargets and Therapy

\author{
Chong Qu ${ }^{1, *}$ \\ Chunmei Dai ${ }^{2, *}$ \\ Yahua Guo ${ }^{3}$ \\ Rui Qin ${ }^{3}$ \\ Junbao $\mathrm{Liu}^{3}$
}

'Department of Neurosurgery, China-Japan Union Hospital of Jilin University, Changchun 130033, Jilin, People's Republic of China; ${ }^{2}$ Department of School Hospital, Changchun University of Chinese Medicine, Changchun I 30033, Jilin, People's Republic of China; ${ }^{3}$ Department of Obstetrics and Gynaecology, China-Japan Union Hospital of Jilin University, Changchun 130033, Jilin,

People's Republic of China

*These authors contributed equally to this work

\begin{abstract}
Objective: This study aims to investigate the functional role of long noncoding RNA SNHG15 in epithelial ovarian cancer (EOC).

Materials and methods: The expression of SNHG15 was measured in EOC cells and tissues using qRT-PCR. The correlation of SNHG15 expression and the clinicopathological characters was statistically analyzed. The prognosis of patients with different clinical features in the high/low SNHG15 expression groups were calculated. Moreover, univariate and multivariate Cox regression analyses were performed to identify the risk factors for poor overall survival (OS) and progression-free survival (PFS). The effect of SNHG15 on the migration and invasion was evaluated using Transwell and Matrigel, respectively. The proliferation ability of EOC cells was tested using colony formation and MTT assay. The influence of SNHG15 on the cisplatin resistance was detected by measuring cell inhibition rate and cell viability.

Results: SNHG15 was upegulated in EOC cells and tissues. High SNHG15 expression was correlated with EOC progression and predicted poor OS and PFS in different subgroups of EOC patients. Moreover, multivariate Cox regression analysis defined high SNHG15 expression as an independent risk factor for poor OS and PFS. Furthermore, functional assays showed that the overexpression of SNHG15 promoted migration and invasion, while the loss of SNHG15 suppressed migration and invasion. Furthermore, the proliferation of EOC cells was improved after the ectopic expression of SNHG15, which was suppressed with SNHG15 deficiency. In addition, cisplatin-resistant EOC cells were established for detecting the effect of SNHG15 on EOC chemoresistance. The results showed that cisplatin-resistant EOC cells exhibited much higher levels of SNHG15 expression than controls, and SNHG15 contributed to the chemoresistance of EOC cells.
\end{abstract}

Conclusion: This study confirms that SNHG15 contributes to the migration, invasion, proliferation, and chemoresistance of EOC. SNHG15 may serve as a potential therapeutic target and prognostic biomarker of EOC patients.

Keywords: noncoding RNA, ovarian cancer, proliferation, metastasis, chemoresistance

\section{Introduction}

Ovarian cancer (OC) accounts for 5\% malignancies in female patients and is the fifth leading cause of death due to cancer in women. ${ }^{1}$ Among the diverse histological subtypes of OC, epithelial OC (EOC) accounts for $\sim 85 \%-90 \%$ of all types of OC. Despite years of developing aggressive surgical techniques and novel adjuvant treatment strategies, EOC remains the one of the most lethal gynecologic malignancies. Current primary treatment strategies for EOC are mainly surgical resection and systemic platinum-based chemotherapy. Unfortunately, $\sim 80 \%$ of patients will experience 
a recurrence within 24 months after completing primary therapy. ${ }^{2,3}$ Furthermore, no reliable method or biomarker currently exists for the prediction of prognosis, which makes it rather difficult to administer individual treatment before recurrence. Not surprisingly, the prognosis for patients with recurrence is poor. Therefore, new methods for the prediction of prognosis and new therapeutic strategies are urgently needed.

The number of long noncoding RNAs (lncRNAs), transcripts longer than 200 nucleotides that do not harbor proteincoding signatures, has exploded in recent years. ${ }^{4}$ Currently, it has been identified that lncRNAs have myriad molecular functions across many cellular pathways and processes, including oncogenic signaling and are likely to serve as the basis for many clinical applications in oncology.$^{5-7}$ When dysregulated, numerous lncRNAs are involved in EOC initiation, development, progression, and chemoresistance. ${ }^{8}$ The involvement of IncRNAs in different EOC stages with various modes of action provides the opportunity to intervene at specific points in EOC development. ${ }^{9}$ Therefore, lncRNAs are suggested to be potential prognostic biomarkers and therapeutic targets in EOC patients. LncRNA SNHG15, located on chromosome $7 \mathrm{p} 13$, has been reported as an oncogene in various types of cancer, such as gastric cancer, ${ }^{10}$ glioma, ${ }^{11}$ non-small-cell lung cancer, ${ }^{12}$ osteosarcoma, ${ }^{13}$ colorectal cancer, ${ }^{14}$ and breast cancer. ${ }^{15}$ However, the clinical significance and functional role of SNHG15 in EOC has not yet been identified.

This study aimed to investigate the role of SNHG15 in EOC. The expression of SNHG15 in EOC cells and tissues was detected, and the relationships between SNHG15 expression and the clinicopathological features of EOC patients were analyzed. The prognostic value of SNHG15 in different subgroups of EOC patients was defined. Furthermore, the effect of SNHG15 on the migration, invasion, proliferation, and chemoresistance of EOC cells was also explored.

\section{Materials and methods}

\section{Cell culture}

The human EOC cell lines (HO89110, OVCAR3, IGROV1, OV90, A2780, and SKOV3) and normal human ovarian surface epithelial (HOSE) cell line were purchased from the Cell Bank of Type Culture Collection, Chinese Academy of Science (Shanghai, People's Republic of China). The cell lines were tested 1 month before the experiments for authentication via methods including morphologic analysis, growth curve analysis, and mycoplasma detection. HO89110 cells and OVCAR3 cells were maintained in DMEM (Invitrogen, Carlsbad, CA, USA), whereas the others were maintained in
RPMI-1640 medium (Invitrogen) supplemented with 10\% FBS (Invitrogen), 2 mM glutamine (Sigma-Aldrich, St. Louis, MO, USA), $100 \mathrm{mg} / \mathrm{mL}$ penicillin (Sigma-Aldrich), and $100 \mathrm{mg} / \mathrm{mL}$ streptomycin (Sigma-Aldrich), and incubated at $37^{\circ} \mathrm{C}$ in a humidified atmosphere with $5 \% \mathrm{CO}_{2}$. SNHG15 siRNAs (si-1, si-2, si-3, and si-4), ectopic expression plasmid (pcDNA3.1-SNHG15), nontargeting control siRNAs (siNC), and empty vector (pcDNA3.1-Vector) were all purchased from GenePharma Co. (Shanghai, People's Republic of China). Transfection was conducted using Lipofectamine 2000 transfection reagent (Invitrogen) according to the manufacturer's instructions.

\section{Tissue collection}

The current study included 182 primary EOC tissues and paired tumor adjacent tissues that were surgically resected between 2010 and 2014 at the China-Japan Union Hospital of Jilin University. The EOC tissues and paired tumor adjacent tissues were confirmed by pathological results. Collection and preservation of the tissues were strictly carried out. Immediately following surgical removal, the tissue samples were frozen in liquid nitrogen and stored at $-80^{\circ} \mathrm{C}$ until use. Patients with two or more malignancies or accepted neoadjuvant therapies were excluded from the study. Clinical and pathological features of the patients are depicted in Table 1. Type I cancers were low grade with a serous, mucinous, endometrioid, or clear-cell histotype; Type II cancers were high grade with a serous, endometrioid,

Table I Correlation between SNHGI5 expression and EOC clinicopathological features

\begin{tabular}{|c|c|c|c|}
\hline Parameters & $\begin{array}{l}\text { No of } \\
\text { patients } \\
(n=\mid 82)\end{array}$ & $\begin{array}{l}\text { SNHGI5 } \\
\text { high/low } \\
(73 / 109)\end{array}$ & $P$-value \\
\hline Age & & & 0.977 \\
\hline$<60$ years & 85 & $34 / 51$ & \\
\hline$\geq 60$ years & 97 & $39 / 58$ & \\
\hline CAI 25 & & & $0.08 I$ \\
\hline$<500 \mathrm{U} / \mathrm{mL}$ & 104 & $36 / 68$ & \\
\hline$\geq 500 \mathrm{U} / \mathrm{mL}$ & 78 & $37 / 41$ & \\
\hline Cancer type & & & 0.001 \\
\hline Type I & 94 & $27 / 67$ & \\
\hline Type II & 88 & $46 / 42$ & \\
\hline Ascites & & & 0.043 \\
\hline$<100 \mathrm{~mL}$ & 111 & $38 / 73$ & \\
\hline$\geq 100 \mathrm{~mL}$ & 71 & $35 / 36$ & \\
\hline FIGO stage & & & 0.049 \\
\hline I/II & 41 & $11 / 30$ & \\
\hline III/IV & 141 & $62 / 79$ & \\
\hline
\end{tabular}

Abbreviations: EOC, epithelial ovarian cancer; FIGO, International Federation of Gynaecology and Obstetrics. 
or undifferentiated histotype. ${ }^{16}$ International Federation of Gynaecology and Obstetrics (FIGO) OC staging system was used for clinical staging. All included patients gave written consent for tissue use in research. The ethics was conducted in accordance with the Declaration of Helsinki. This study was approved by the Ethics Committee of the China-Japan Union Hospital of Jilin University.

\section{qRT-PCR assay}

Total RNA was isolated from EOC cells and human tissues using TRIzol reagent (Invitrogen). Total RNA (1 mg) was reverse transcribed using a cDNA synthesis kit (Roche Diagnostics, Indianapolis, IN, USA) with anchored-oligo (dT) 18 primers. Quantitative PCR was performed under standard conditions using an Eppendorf real-time PCR machine and an SYBR Green I Master Kit (Roche Diagnostics). The reactions were performed at $95^{\circ} \mathrm{C}$ for 10 minutes to activate the polymerase, followed by 40 cycles at $95^{\circ} \mathrm{C}$ for 5 seconds, $55^{\circ} \mathrm{C}$ for 10 seconds, and $72^{\circ} \mathrm{C}$ for 20 seconds. GAPDH was used as endogenous controls. The relative expression level was calculated using the $2^{-\Delta \Delta \mathrm{Ct}}$ method. The primer sequences used are as follows: SNHG15 (sense: 5'-CAACCATAGCGGTGCAACTGTGC-3', antisense: 3'-GGCTGAACCAAGTTGCAAGTCATG-5'); GAPDH (sense: 5'-CAGTGCCAGCCTCGTCTAT-3', antisense: 3'-AGGGGCCATCCACAGTCTTC-5'). The experiments were repeated three times in triplicate.

\section{In vitro cell migration and invasion assay}

The migration and invasion abilities of EOC cells with SNHG15 deficiency or ectopic expression were assessed by a Transwell assay and a Matrigel assay using Transwell chambers (pore size, $8 \mu \mathrm{m}$; BD Biosciences). Cells $\left(5 \times 10^{4}\right)$ suspended in serum-free medium were seeded in the upper chamber (precoated with Matrigel in the Matrigel assay), while the lower chamber was filled with medium containing $20 \%$ FBS. After 24 hours of incubation, cells remaining on top of the membrane were removed using a cotton swab. Migrated/invaded cells on the lower surface of the membrane were stained with Giemsa, photographed with a light microscope (Olympus Corp., Tokyo, Japan), and counted. The experiments were repeated three times in triplicate.

\section{Colony formation assay}

The colony formation assay was performed to evaluate the proliferation ability of EOC cells. Briefly, EOC cells (500 cells per well) were incubated in 6-well plates, and the medium was changed every 3 days. Two weeks later, formed colonies were fixed with methanol for 15 minutes and stained with $0.1 \%$ crystal violet for another 15 minutes. Colonies with a diameter greater than $1 \mathrm{~mm}$ were counted under a light microscope (Olympus Corp.). The experiments were repeated three times in triplicate.

\section{MTT assay}

The MTT assay was conducted to assess the proliferation ability and cisplatin resistance. EOC cells (3,000 cells per well) were seeded in 96-well plates. After transfection, cells were incubated with $20 \mu \mathrm{L}$ MTT $(5 \mathrm{mg} / \mathrm{mL})$ in each well at the indicated time points $(0,24,48,72$, and 96 hours) for 4 hours. Then, dimethyl sulfoxide (Sigma) was added (150 $\mu \mathrm{L} /$ well) to each well to dissolve the formazan crystals followed by agitation of the plates for 10 minutes. OD was measured at $490 \mathrm{~nm}$ on a microplate reader (Molecular Devices, Sunnyvale, CA, USA). The experiments were repeated three times in triplicate.

To investigate the effect of cisplatin on cell proliferation, cisplatin $(0.1 \mathrm{~mL}, 0-80 \mu \mathrm{M})$ was added to the culture medium, and cells were incubated for another 48 hours. Then the MTT assay was conducted to analyze the absorbance. The results of the OD values were calculated to obtain inhibition rates to express the effect of cisplatin. The experiments were repeated three times in triplicate.

\section{Establishment of cisplatin resistant cells}

EOC cells, A2780 and SKOV3, were treated with cisplatin $(5 \mu \mathrm{M})$ for 3 hours and then allowed to recover for 2 weeks. Surviving cells were passaged and subjected to the next round of treatment. Sequentially increasing concentrations $(5 \mu \mathrm{M})$ of cisplatin were used to select resistant cells and finally maintained at $35 \mu \mathrm{M}$. The obtained cisplatin-resistant A2780 cells and SKOV3 cells were named A2780-R and SKOV3-R, respectively.

\section{Statistical analysis}

The statistical calculations were performed with GraphPad Prism 6.0 for Windows (GraphPad Software, La Jolla, CA, USA). The results are shown as the mean $\pm \mathrm{SD}$. The statistical significance of the differences among different groups was calculated using a two-tailed Student's $t$-test or chi-squared test. Kaplan-Meier plots for overall survival (OS) and progression-free survival (PFS) were plotted, and analysis was done using the log-rank test. Univariate and multivariate Cox regression analyses were conducted to analyze the risk factors for poor prognosis. A $P$-value of less than 0.05 was considered to be statistically significant. 


\section{Results}

\section{SNHGI 5 is upregulated in EOC and is associated with cancer progression}

To examine the role of SNHG15 in EOC, the expression of SNHG15 was measured in HOSE and six EOC cell lines. Interestingly, qRT-PCR results found that SNHG15 was upregulated in six EOC cell lines relative to HOSE (Figure 1A). Further detection of the SNHG15 expression level was conducted in $182 \mathrm{EOC}$ tissues and paired tumor adjacent tissues. As expected, EOC tissues displayed much higher level of SNHG15 than paired tumor adjacent tissues (Figure 1B). Furthermore, patients with Type II cancers showed higher SNHG15 levels than patients with Type I cancers (Figure 1C). For a better understanding of the clinical significance of SNHG15 in EOC, the involved patients were divided into two groups, the low SNHG15 expression group ( $\mathrm{n}=109)$ and the high SNHG15 expression group $(n=73)$, with the mean SNHG15 expression level serving as the cutoff value. As shown in Table 1, the differences in the clinicopathological features between the low SNHG15 expression group and the high SNHG15 expression group were statistically evaluated. High SNHG15 expression was revealed to correlate with cancer type $(P=0.001)$, ascites ( $P=0.043)$, and FIGO stage ( $P=0.049)$ (Table 1). Therefore, the overexpression of SNHG15 is associated with the clinical progression of EOC.

\section{SNHGI 5 predicts poor prognosis in different subgroups of EOC patients}

OS and PFS are two fundamental parameters evaluating the prognosis of cancer patients. The prediction value of SNHG15 on OS and PFS was explored in different subgroups of EOC patients. The results showed that patients with low SNHG15 expression had a significantly improved OS rate (Figure 2A, left) and PFS rate (Figure 2A, right) than patients with high SNHG15 expression. For patients with Type I cancers, high SNHG15 expression reduced the OS rate compared with low SNHG15 expression, although no statistical

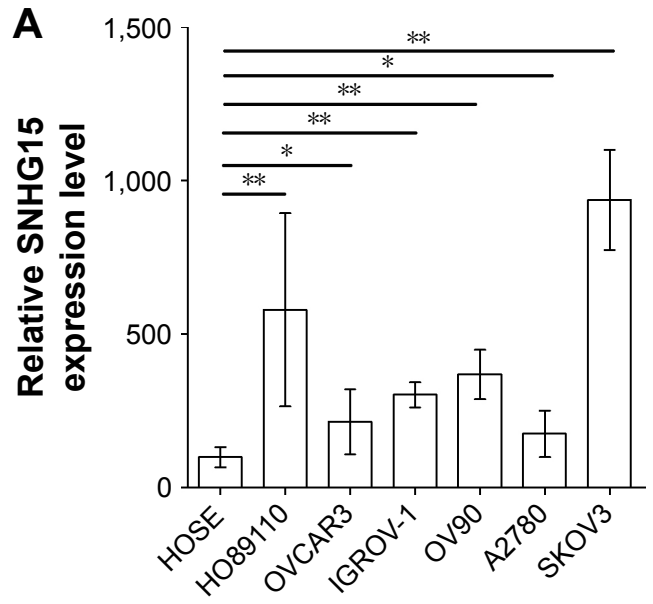

C

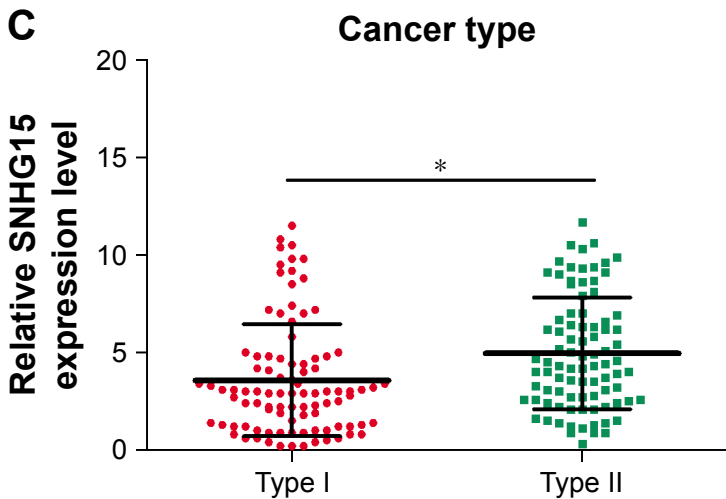

B

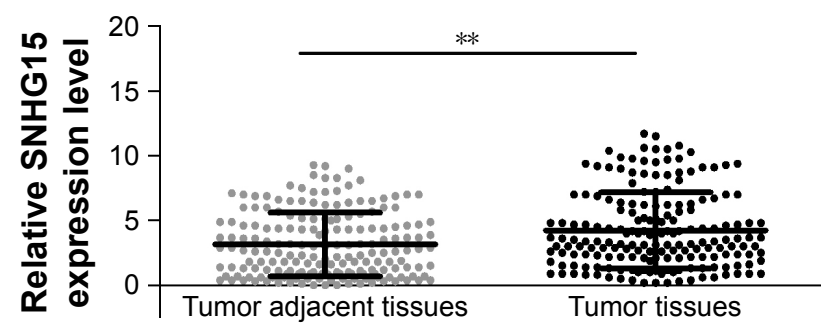

Figure I SNHGI5 is upregulated in EOC.

Notes: (A) Expression of SNHGI5 was measured in six EOC cell lines (HO89I I0, OVCAR3, IGROV-I, OV90, A2780, and SKOV3) and normal cell line human ovarian surface epithelial (HOSE) by qRT-PCR assay. (B) Expression of SNHGI5 was detected in EOC tissues and paired tumor adjacent tissues ( $\mathrm{n}=182$ ) by a qRT-PCR assay. (C) Expression of SNHGI5 in Type I and Type II disease was detected by a qRT-PCR assay. $* P<0.05$ and $* * P<0.0$ I.

Abbreviation: EOC, epithelial ovarian cancer. 
A

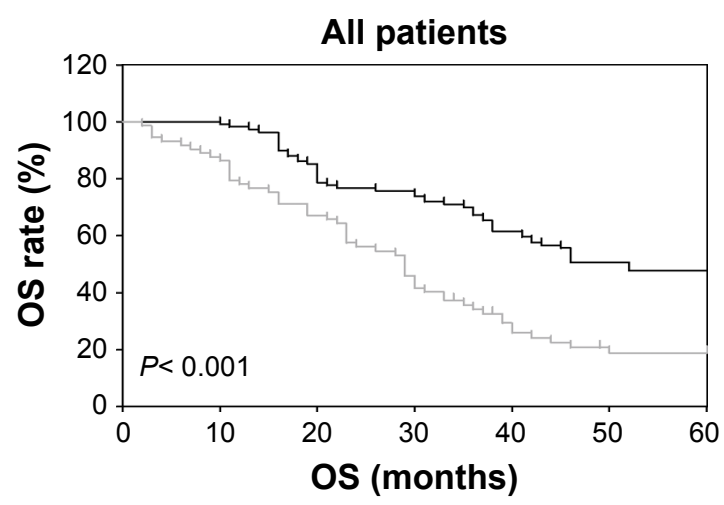

B

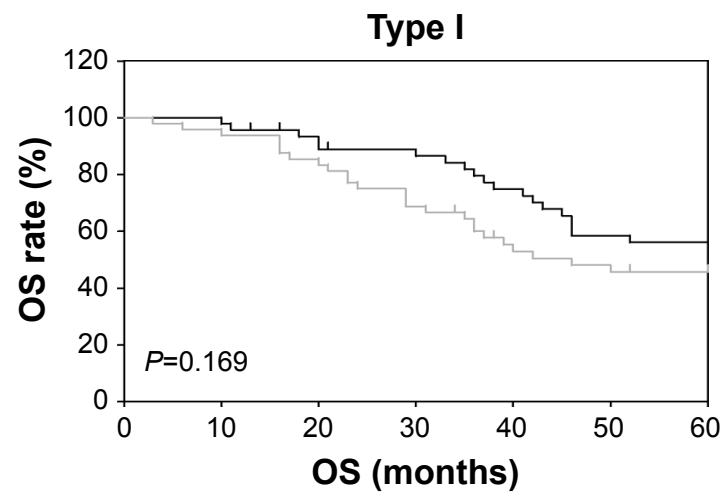

C

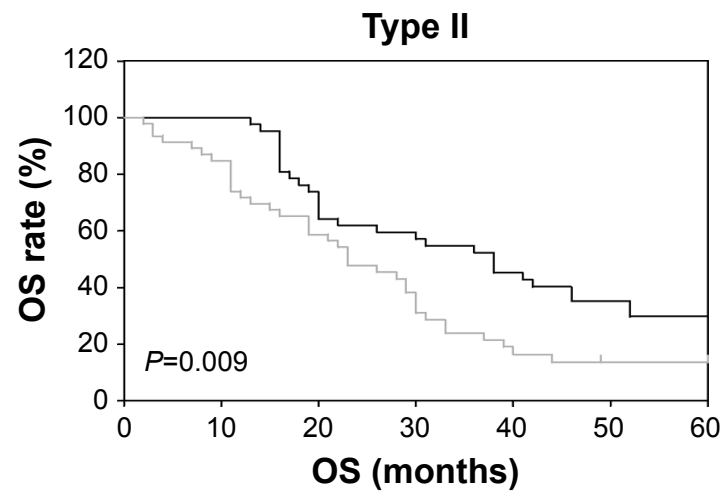

All patients

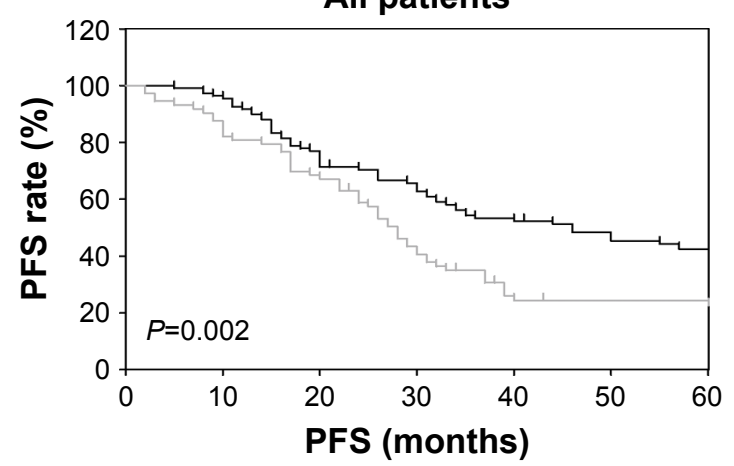

Type I

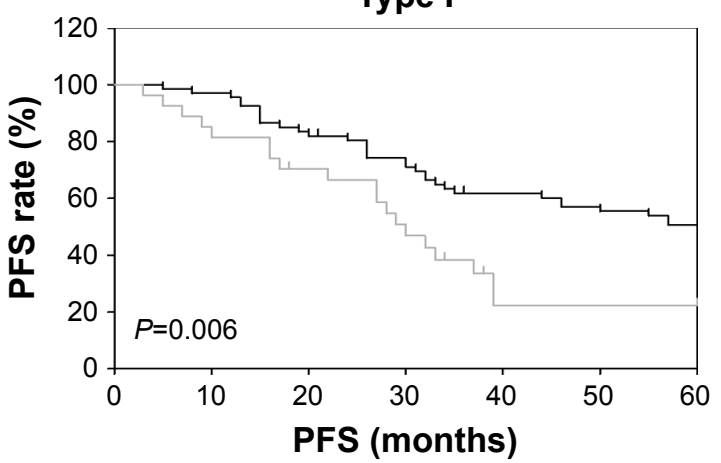

Type II

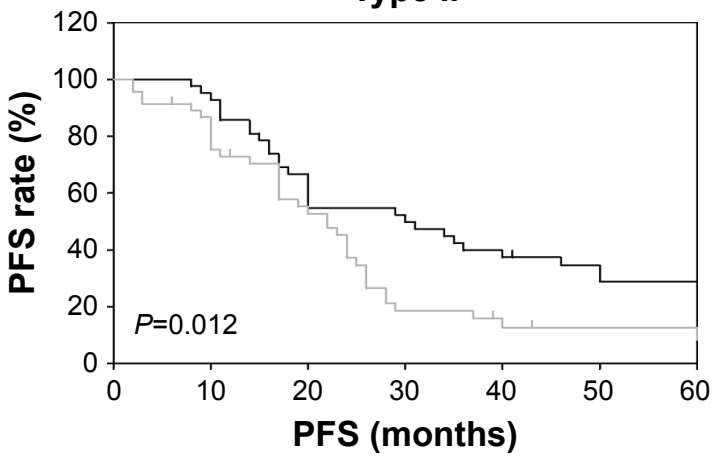

+ Low SNHG15 + High SNHG15

Figure 2 SNHGI 5 predicts poor prognosis in different subgroups of EOC patients.

Notes: (A) The OS and PFS rate of EOC patients with low SNHGI5 expression and high SNHGI5 expression were calculated. (B) The OS rate and PFS rate of patients with Type I cancers in the low SNHGI 5 expression group and the high SNHGI5 expression group were calculated. (C) The OS rate and PFS rate of patients with Type II cancers in the low SNHGI 5 expression group and the high SNHGI5 expression group were calculated.

Abbreviations: EOC, epithelial ovarian cancer; OS, overall survival; PFS, progression-free survival.

significance was found (Figure 2B, left). However, the PFS rate was significantly elevated in the low SNHG15 expression group compared with the high SNHG15 expression group (Figure 2B, right). Moreover, in the subgroup of patients with Type II disease, high SNHG15 expression indicated poor OS (Figure 2C, left) and PFS (Figure 2C, right).

Furthermore, the risk factors for poor OS and PFS were investigated with univariate and multivariate Cox regression analyses. The results discovered that Type II cancers
$(\mathrm{HR}=2.430,95 \% \mathrm{CI}=1.656-3.565, P<0.001)$, advanced FIGO stage $(\mathrm{HR}=2.223,95 \% \mathrm{CI}=1.354-2.651, P=0.002)$, and high SNHG15 expression $(\mathrm{HR}=1.151,95 \% \mathrm{CI}=1.086-1.220$, $P<0.001$ ) were risk factors for poor OS (Table 2). Further analysis of these factors with multivariate Cox regression analysis revealed that Type II cancers $(\mathrm{HR}=1.960$, 95\% CI=1.319-2.915, $P=0.001)$, advanced FIGO stage $(\mathrm{HR}=1.424,95 \% \mathrm{CI}=1.108-3.071, P=0.019)$, and high SNHG15 expression $(\mathrm{HR}=1.136,95 \% \mathrm{CI}=1.069-1.208$, 
Table 2 Univariate and multivariate analysis of clinicopathological features for overall survival of EOC patients

\begin{tabular}{|c|c|c|c|c|c|c|}
\hline \multirow[t]{2}{*}{ Parameters } & \multicolumn{3}{|c|}{ Univariate analysis } & \multicolumn{3}{|c|}{ Multivariate analysis } \\
\hline & HR & $95 \% \mathrm{Cl}$ & $P$-value & HR & $95 \% \mathrm{Cl}$ & $P$-value \\
\hline Age: $\geq 60$ years vs $<60$ years & 0.810 & $\begin{array}{l}0.558 \\
-1.176\end{array}$ & 0.268 & & & \\
\hline CAI25: $<500 \mathrm{U} / \mathrm{mL}$ vs $\geq 500 \mathrm{U} / \mathrm{mL}$ & 1.194 & $\begin{array}{l}0.819 \\
-1.741\end{array}$ & 0.356 & & & \\
\hline Cancer type: Type I vs Type II & 2.430 & \begin{tabular}{|l|l|}
1.656 \\
-3.565 \\
\end{tabular} & $<0.00 \mathrm{I}$ & 1.960 & $\begin{array}{l}1.319 \\
-2.915\end{array}$ & 0.001 \\
\hline Ascites: $<100 \mathrm{~mL}$ vs $\geq 100 \mathrm{~mL}$ & 1.322 & \begin{tabular}{|l|l}
0.901 \\
-1.938 \\
\end{tabular} & 0.153 & & & \\
\hline FIGO stage: III/IV vs I/II & 2.223 & \begin{tabular}{|l|}
.354 \\
-2.651
\end{tabular} & 0.002 & 1.424 & $\begin{array}{l}1.108 \\
-3.071\end{array}$ & 0.019 \\
\hline SNHGI5: high vs low & 1.151 & $\begin{array}{l}1.086 \\
-1.220\end{array}$ & $<0.001$ & 1.136 & $\begin{array}{l}1.069 \\
-1.208\end{array}$ & $<0.001$ \\
\hline
\end{tabular}

Abbreviations: EOC, epithelial ovarian cancer; FIGO, International Federation of Gynaecology and Obstetrics.

$P<0.001$ ) are independent risk factors for poor OS (Table 2). Furthermore, a similar analysis was performed to identify the risk factors of poor PFS, and high SNHG15 expression $(\mathrm{HR}=1.120,95 \% \mathrm{CI}=1.056-1.189, P<0.001)$ was revealed as an independent risk factor for poor PFS (Table 3). Collectively, SNHG15 may be a promising prognostic biomarker for EOC patients with different histological types.

\section{SNHG 5 promotes the migration, invasion, and proliferation of EOC cells}

To further verify the function of SNHG15 in EOC, SNHG15 was silenced with four siRNAs and overexpressed with pcDNA3.1-SNHG15 in SKOV3 cells and A2780 cells, respectively. The expression of SNHG15 in EOC cells after transfection was measured by a qRT-PCR assay (Figure 3A). siRNAs with relatively high efficacies (si-2 and si-4) were selected for further experiments. The Transwell and Matrigel assays were adopted to investigate the effect of SNHG15 on the migration and invasion of EOC cells, respectively. Intriguingly, the overexpression of SNHG15 in A2780 cells markedly promoted the migration (Figure $3 \mathrm{~B}$ ) and invasion (Figure 3C) of EOC cells. Accordingly, interference with SNHG15 in SKOV3 cells notably suppressed the migration (Figure 3D) and invasion (Figure 3E) of EOC cells. Taken together, SNHG15 upregulation promotes the migration and invasion of EOC cells.

The proliferation of EOC cells after transfection was determined by a colony formation assay and a MTT assay. It was shown that the ectopic expression of SNHG15 increased the colony number (Figure 4A) and OD value (Figure 4B) of A2780 cells. The loss of SNHG15 decreased the proliferation of SKOV3 cells (Figure 4C and D).

Table 3 Univariate and multivariate analysis of clinicopathological features for progression-free survival of EOC patients

\begin{tabular}{|c|c|c|c|c|c|c|}
\hline \multirow[t]{2}{*}{ Parameters } & \multicolumn{3}{|c|}{ Univariate analysis } & \multicolumn{3}{|c|}{ Multivariate analysis } \\
\hline & HR & $95 \% \mathrm{Cl}$ & $P$-value & HR & $95 \% \mathrm{Cl}$ & $P$-value \\
\hline Age: $\geq 60$ years vs $<60$ years & 0.759 & $\begin{array}{l}0.527 \\
-1.094\end{array}$ & 0.140 & & & \\
\hline CAI25: $<500 \mathrm{U} / \mathrm{mL}$ vs $\geq 500 \mathrm{U} / \mathrm{mL}$ & 1.103 & $\begin{array}{l}0.762 \\
-1.597\end{array}$ & 0.603 & & & \\
\hline Cancer type: Type I vs Type II & 2.054 & $\begin{array}{l}1.418 \\
-2.974\end{array}$ & $<0.00 \mathrm{I}$ & 1.721 & $\begin{array}{l}1.175 \\
-2.520\end{array}$ & 0.005 \\
\hline Ascites: $<100 \mathrm{~mL}$ vs $\geq 100 \mathrm{~mL}$ & 1.371 & $\begin{array}{l}0.944 \\
-1.990\end{array}$ & 0.097 & & & \\
\hline FIGO stage: III/IV vs I/II & 1.911 & $\begin{array}{l}1.199 \\
-3.046\end{array}$ & 0.006 & 1.659 & $\begin{array}{l}1.031 \\
-2.670\end{array}$ & 0.037 \\
\hline SNHGI5: high vs low & 1.134 & $\begin{array}{l}1.072 \\
-1.200\end{array}$ & $<0.001$ & 1.120 & $\begin{array}{l}1.056 \\
-1.189\end{array}$ & $<0.001$ \\
\hline
\end{tabular}

Abbreviations: EOC, epithelial ovarian cancer; FIGO, International Federation of Gynaecology and Obstetrics. 
A

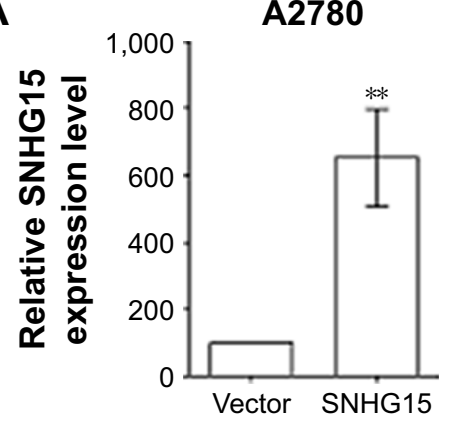

B

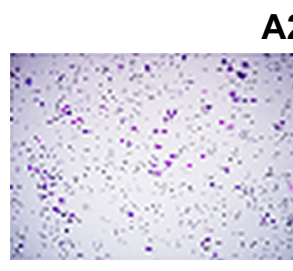

Vector

C

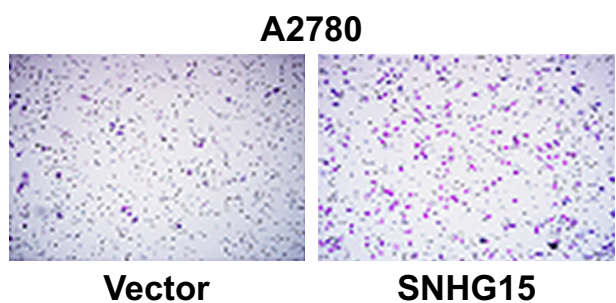

D

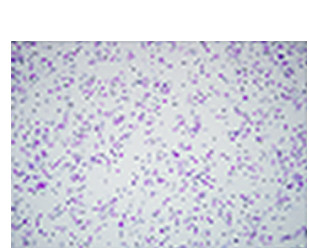

siNC
A2780

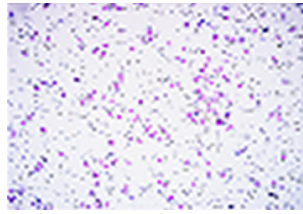

SNHG15

SNHG15
SKOV3

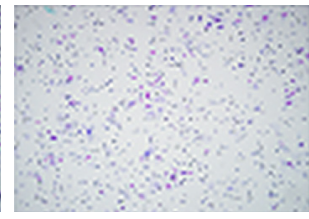

si-2
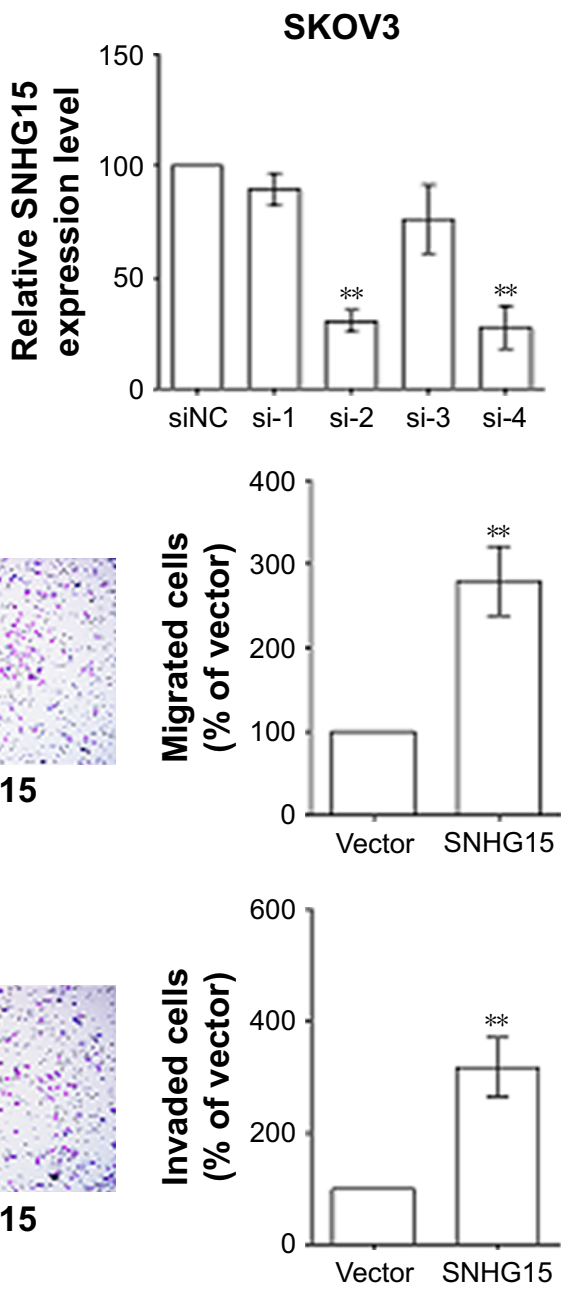

E

\section{SKOV3}

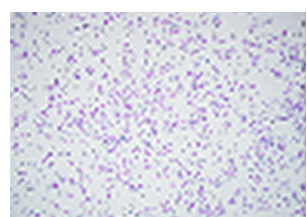

siNC
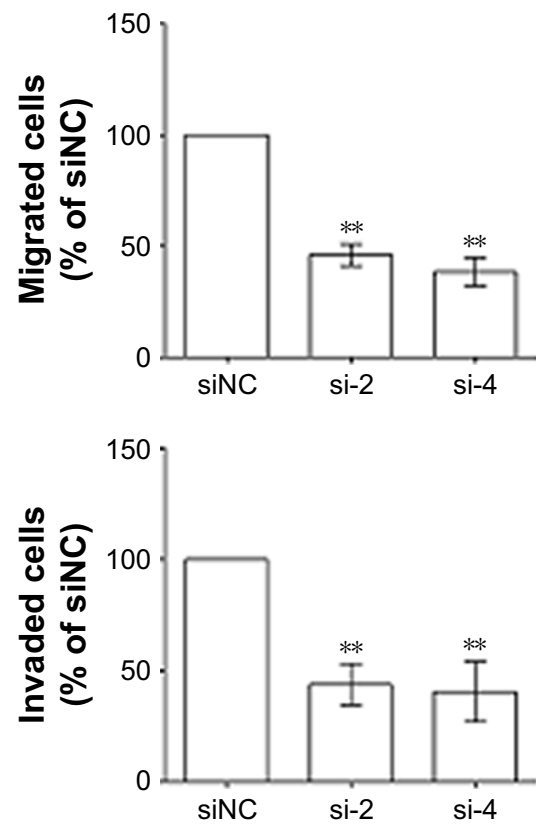

Figure 3 SNHGI 5 promotes the migration and invasion of EOC cells.

Notes: (A) Expression of SNHGI5 in A2780 cells with SNHG overexpression and SKOV3 cells with SNHGI 5 deficiency was detected by a qRT-PCR assay. (B) Migration ability of A2780 cells with SNHG 5 overexpression was evaluated by a Transwell assay. (C) Invasion ability of A2780 cells with SNHG I5 overexpression was evaluated by a Matrigel assay. (D) Migration ability of SKOV3 cells with SNHGI 5 deficiency was evaluated by a Transwell assay. (E) Invasion ability of SKOV3 cells with SNHGI 5 deficiency was evaluated by a Matrigel assay. $* * P<0.01$.

Abbreviations: EOC, epithelial ovarian cancer; siNC, nontargeting control siRNAs. 
A

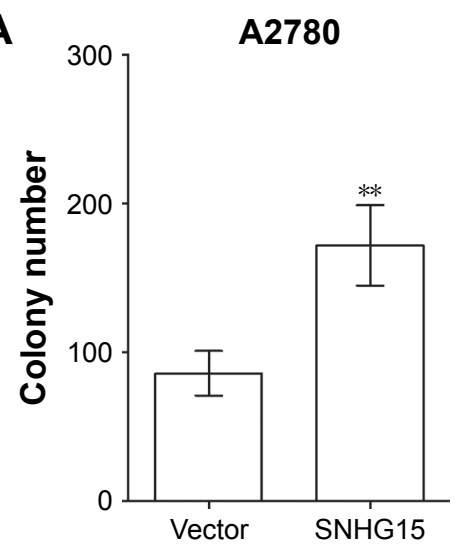

C

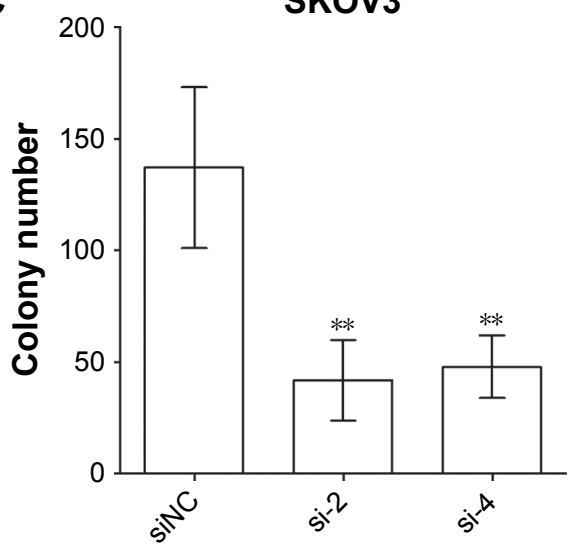

B

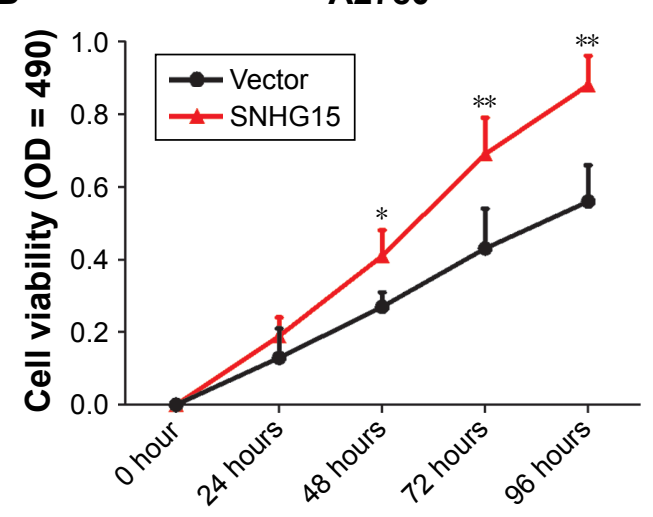

D

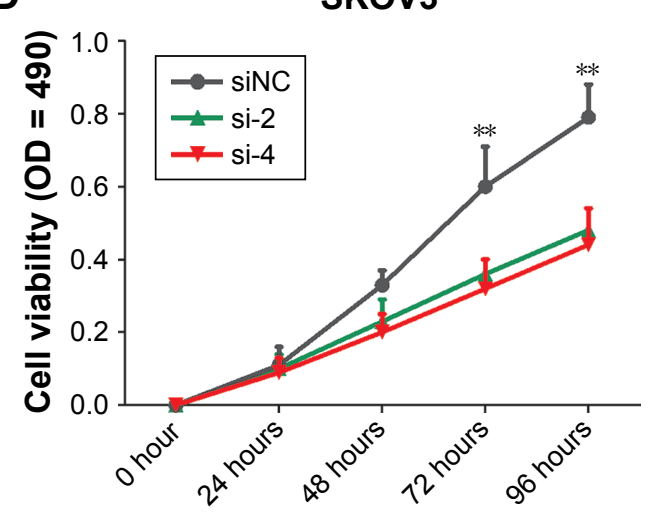

Figure 4 SNHGI 5 promotes the proliferation of EOC cells.

Notes: (A) The colony formation assay was used to determine the proliferation ability of A2780 cells with SNHGI5 overexpression. (B) The MTT assay was performed to investigate the proliferation ability of A2780 cells with SNHGI 5 overexpression. (C) The colony formation assay was adopted to determine the proliferation ability of SKOV3 cells with SNHGI 5 deficiency. (D) The MTT assay was performed to investigate the proliferation ability of SKOV 3 cells with SNHGI 5 deficiency. $* P<0.05$ and $* * P<0.01$.

Abbreviations: EOC, epithelial ovarian cancer; siNC, nontargeting control siRNAs.

These results indicate that SNHG15 accelerates the proliferation of EOC cells.

\section{SNHGI 5 contributes to the chemoresistance of EOC cells}

Chemoresistance is a primary contributing factor leading to the final relapse of EOC patients. To illuminate the function of SNHG15 on chemoresistance of EOC cells, cisplatin-resistant cells (A2780-R and SKOV3-R) were established. The inhibition rates of cisplatin on A2780-R cells (Figure 5A) and SKOV3-R (Figure 5B) cells were greatly reduced compared with A2780 cells and SKOV3 cells, respectively. Noticeably, A2780-R cells and SKOV3-R cells had much higher SNHG15 levels than A2780 cells and SKOV3 cells, respectively (Figure 5C and D), which implicated the function of SNHG15 on cisplatin resistance of EOC cells. In addition, the inhibition rate of cisplatin on A2780 cells with SNHG15 overexpression was decreased (Figure 5E), and SNHG15 deficiency in SKOV3 cells increased the inhibition rate of cisplatin significantly (Figure 5F). Therefore, SNHG15 contributes to the chemoresistance of EOC cells.

\section{Discussion}

lncRNAs are considered important factors in several histological types of OC. ${ }^{9}$ Different variants of lncRNAs have been revealed to be correlated with the risk and functionality of EOC. ${ }^{17,18}$ For example, the genotyping analyses of lncRNA HOX transcript antisense intergenic RNA (HOTAIR) indicated that rs4759314 and rs7958904 were significantly associated with epithelial EOC susceptibility, which suggested that HOTAIR variants could be a useful biomarker for the predisposition to epithelial EOC and for the early diagnosis of the disease. ${ }^{17}$ Another study by Qiu et a $1^{18}$ reported that HOTAIR rs920778 polymorphism influenced EOC susceptibility and prognosis. The involvement of IncRNAs in EOC has been widely reported, and current evidence demonstrates that lncRNAs could modulate EOC initiation, development, progression, and chemoresistance. 
A
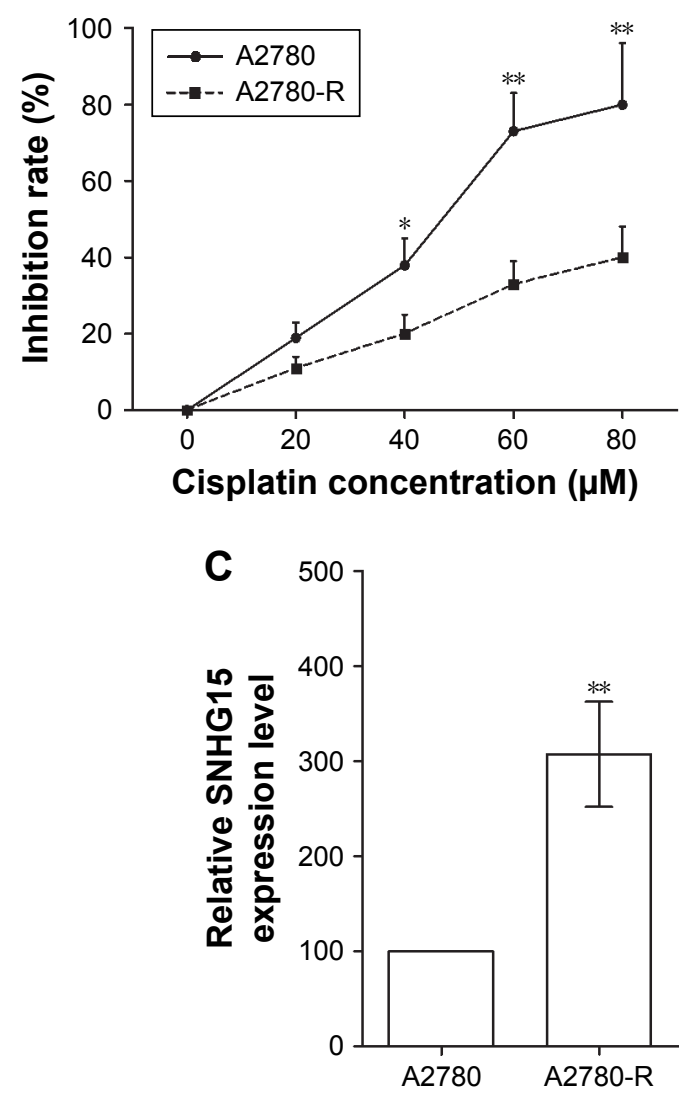

E

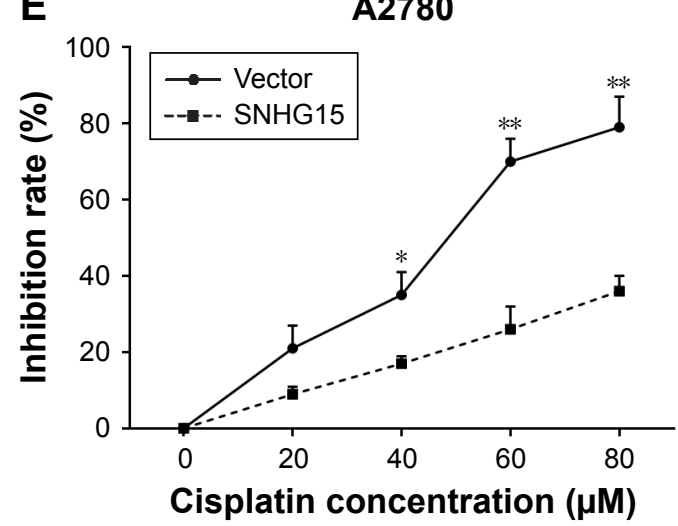

B
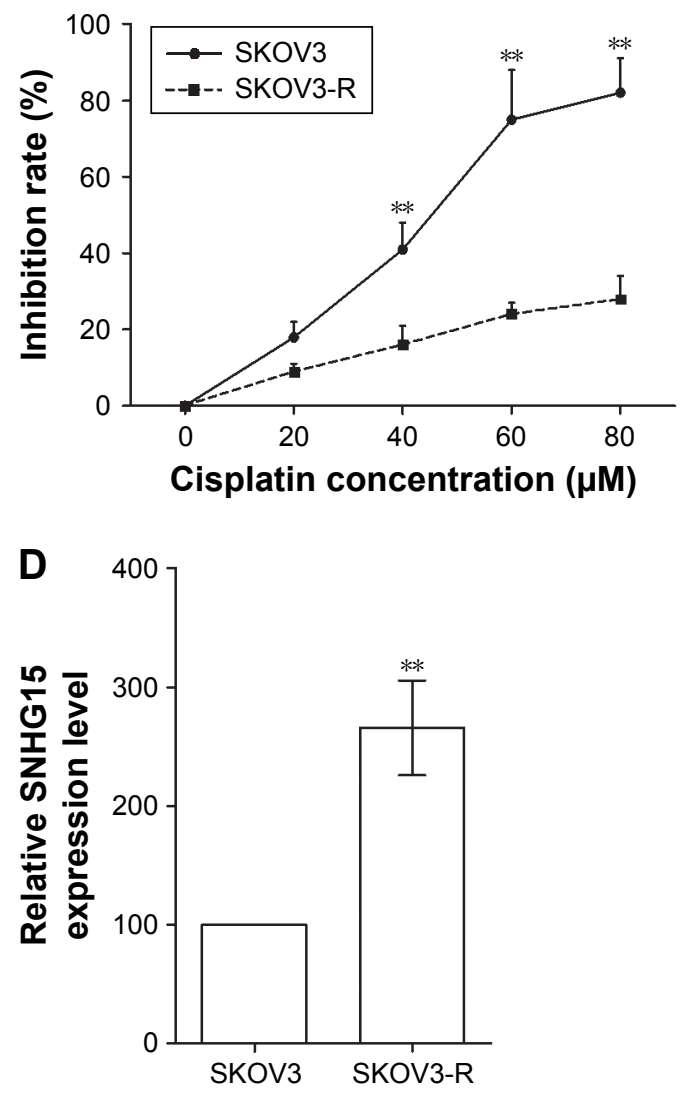

$\mathbf{F}$

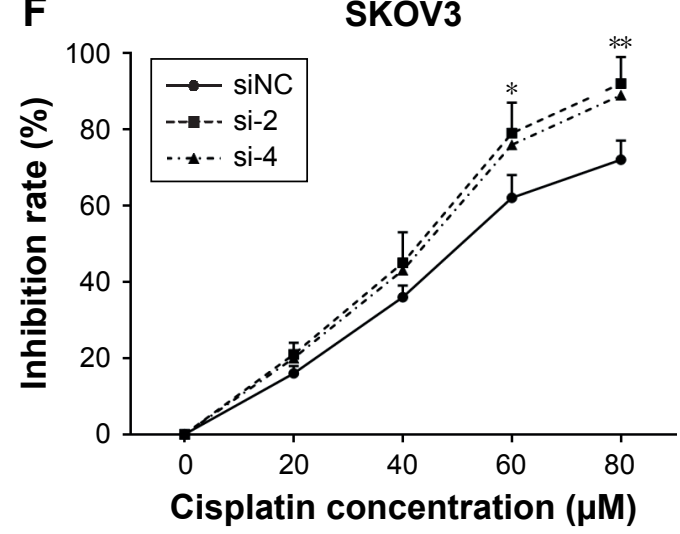

Figure 5 SNHGI 5 contributes to the chemoresistance of EOC cells.

Notes: (A) The inhibition rate of cisplatin on A2780 cells and A2780-R cells were detected by MTT assay. (B) The inhibition rate of cisplatin on SKOV3 cells and SKOV3-R cells were detected by MTT assay. (C) Expression of SNHGI5 in A2780 cells and A2780-R cells was detected by qRT-PCR assay. (D) Expression of SNHGI5 in SKOV3 cells and SKOV3-R cells was detected by qRT-PCR assay. (E) Inhibition rates of cisplatin on A2780 cells transfected with SNHG 5 overexpression plasmid and empty vector after cisplatin treatment were measured by MTT assay. (F) Inhibition rates of cisplatin on SKOV3 cells transfected with SNHGI5 siRNAs and siNC after cisplatin treatment were measured by MTT assay. $* P<0.05$ and $* * P<0.01$.

Abbreviations: A2780-R, cisplatin-resistant A2780 cells; EOC, epithelial ovarian cancer; SKOV3-R, cisplatin-resistant SKOV3 cells; siNC, nontargeting control siRNAs.

Metastasis-associated lung adenocarcinoma transcript 1 (MALAT1) is one of the major focuses in EOC, and in vivo studies have confirmed that MALAT1 could promote the growth and metastasis of EOC cells. ${ }^{19-22}$ Some other IncRNAs, such as brain cytoplasmic RNA $200,{ }^{23}$ growth arrest-specific $5,{ }^{24}$ colon cancer associated transcript 2
(CCAT2), ${ }^{25}$ and taurine upregulated gene $1^{26}$ have also been revealed to mediate the biology of EOC cells. Furthermore, lncRNAs are considered as potentially novel clinical tools for patient management because they are often tissue-specific and exhibit poor sequence conservation across species. ${ }^{27}$ Several lncRNAs have been identified as 
potential diagnostic and prognostic prediction biomarkers in different EOC histological types, such as MALAT1, ${ }^{19}$ C17orf91, ${ }^{28}$ and CCAT2. ${ }^{25}$ LncRNA-based diagnosis, prognostic prediction, and treatment may shed light on the management of EOC patients.

In 2013, Tani et $\mathrm{al}^{29}$ discovered that SNHG15 was upregulated in HeLa Tet-off cells when exposed to cycloheximide, and the expression of SNHG15 increased in response to increasing concentrations of cycloheximide. Two years later, the same team found that the overexpression of SNHG15 caused a decrease in the cell viability of HEK293 cells, whereas it had no effect on cell proliferation. ${ }^{30}$ Currently, SNHG15 has been reported to be overexpressed and serves as an oncogene in hepatocellular carcinoma, ${ }^{31}$ pancreatic cancer, ${ }^{32}$ glioma, ${ }^{11}$ gastric cancer, ${ }^{10}$ non-small-cell lung cancer, ${ }^{12}$ colon cancer, ${ }^{14}$ breast cancer, ${ }^{15}$ and osteosarcoma. ${ }^{13}$ Functionally, SNHG15 was involved in the proliferation, invasion, migration, chemoresistance, and autophagy of different cancers. ${ }^{12,13,15,33}$ Mechanistically, SNHG15 could exert its functional by sponging miRNAs, such as miR-141, ${ }^{13}$ miR-211-3p, ${ }^{15}$ miR-153, ${ }^{11}$ and miR-486. ${ }^{12}$ Additionally, the RNA immunoprecipitation assay showed that SNHG15 could epigenetically repress the P15 and Kruppel-like factor 2 (KLF2) expression via binding to enhancer of zeste homologue 2 (EZH2) in pancreatic cancer cells. ${ }^{32}$ Chen et $\mathrm{al}^{10}$ reported that $\mathrm{SNHG} 15$ could regulate the expression of MMP2 and MMP9, which promoted cell proliferation and invasion. SNHG15 is involved in almost every aspect of cancer biology; it may be a promising biomarker and therapeutic target in cancer management.

The present study revealed that SNHG15 was overexpressed in EOC cell lines and tissues compared to HOSE cells and paired tumor adjacent tissues, respectively. Further statistical analysis found that high SNHG15 expression was correlated with EOC progression, which indicated its oncogenic role in EOC. Further investigations found that patients with high SNHG15 expression showed poor OS and PFS in different subgroups of EOC patients, and high SNHG15 expression was identified as an independent risk factor for poor OS and PFS. The results suggested the prediction value of SNHG15 for the prognosis of EOC patients. Moreover, the function of SNHG15 was verified in EOC cells, and SNHG15 was demonstrated to promote the migration, invasion, proliferation, and chemoresistance of EOC cells. However, the detailed mechanisms by which SNHG15 exerts its function were not investigated in the present study, and these mechanisms deserve further exploration.

\section{Conclusion}

Our findings present that SNHG15 may be a novel biomarker for prognostic prediction and a potential therapeutic target in $\mathrm{EOC}$.

\section{Disclosure}

The authors report no conflicts of interest in this work.

\section{References}

1. Siegel RL, Miller KD, Jemal A. Cancer statistics, 2016. CA Cancer J Clin. 2016;66(1):7-30.

2. Scalici JM, Arapovic S, Saks EJ, et al. Mesothelium expression of vascular cell adhesion molecule-1 (VCAM-1) is associated with an unfavorable prognosis in epithelial ovarian cancer (EOC). Cancer. 2017; 123(6):977-984.

3. Modugno F, Edwards RP. Ovarian cancer: prevention, detection, and treatment of the disease and its recurrence. Molecular mechanisms and personalized medicine meeting report. Int J Gynecol Cancer. 2012; 22(8):S45-S57.

4. Beermann J, Piccoli MT, Viereck J, Thum T. Non-coding RNAs in development and disease: background, mechanisms, and therapeutic approaches. Physiol Rev. 2016;96(4):1297-1325.

5. Evans JR, Feng FY, Chinnaiyan AM. The bright side of dark matter: IncRNAs in cancer. $J$ Clin Invest. 2016;126(8):2775-2782.

6. Gutschner T, Diederichs S. The hallmarks of cancer: a long non-coding RNA point of view. RNA Biol. 2012;9(6):703-719.

7. Bolha L, Ravnik-Glavač M, Glavač D. Long noncoding RNAs as biomarkers in cancer. Dis Markers. 2017;2017:7243968.

8. Meryet-Figuière M, Lambert B, Gauduchon P, et al. An overview of long non-coding RNAs in ovarian cancers. Oncotarget. 2016;7(28): 44719-44734.

9. Worku T, Bhattarai D, Ayers D, et al. Long non-coding RNAs: the new horizon of gene regulation in ovarian cancer. Cell Physiol Biochem. 2017;44(3):948-966.

10. Chen SX, Yin JF, Lin BC, et al. Upregulated expression of long noncoding RNA SNHG15 promotes cell proliferation and invasion through regulates MMP2/MMP9 in patients with GC. Tumour Biol. 2016; 37(5):6801-6812.

11. Ma Y, Xue Y, Liu X, et al. SNHG15 affects the growth of glioma microvascular endothelial cells by negatively regulating miR-153. Oncol Rep. 2017;38(5):3265-3277.

12. Jin B, Jin H, Wu HB, Xu JJ, Li B. Long non-coding RNA SNHG15 promotes CDK14 expression via miR-486 to accelerate non-small cell lung cancer cells progression and metastasis. J Cell Physiol. 2018; 233(9):7164-7172.

13. Liu K, Hou Y, Liu Y, Zheng J. LncRNA SNHG15 contributes to proliferation, invasion and autophagy in osteosarcoma cells by sponging miR-141. J Biomed Sci. 2017;24(1):46.

14. Jiang $\mathrm{H}, \mathrm{Li} \mathrm{T}$, Qu Y, et al. Long non-coding RNA SNHG15 interacts with and stabilizes transcription factor Slug and promotes colon cancer progression. Cancer Lett. 2018;425:78-87.

15. Kong Q, Qiu M. Long noncoding RNA SNHG15 promotes human breast cancer proliferation, migration and invasion by sponging miR-211-3p. Biochem Biophys Res Commun. 2018;495(2):1594-1600

16. Romero I, Bast RC. Minireview: human ovarian cancer: biology, current management, and paths to personalizing therapy. Endocrinology. 2012;153(4):1593-1602.

17. Wu H, Shang X, Shi Y, et al. Genetic variants of IncRNA HOTAIR and risk of epithelial ovarian cancer among Chinese women. Oncotarget. 2016;7(27):41047-41052

18. Qiu H, Wang X, Guo R, et al. HOTAIR rs 920778 polymorphism is associated with ovarian cancer susceptibility and poor prognosis in a Chinese population. Future Oncol. 2017;13(4):347-355. 
19. Lin Q, Guan W, Ren W, Zhang L, Zhang J, Xu G. MALAT1 affects ovarian cancer cell behavior and patient survival. Oncol Rep. 2018; 39(6):2644-2652.

20. Zhou Y, Xu X, Lv H, et al. The long noncoding RNA MALAT-1 is highly expressed in ovarian cancer and induces cell growth and migration. PLoS One. 2016;11(5):e0155250.

21. Lei R, Xue M, Zhang L, Lin Z. Long noncoding RNA MALAT1regulated microRNA 506 modulates ovarian cancer growth by targeting iASPP. Onco Targets Ther. 2017;10:35-46.

22. Jin Y, Feng SJ, Qiu S, Shao N, Zheng JH. LncRNA MALAT1 promotes proliferation and metastasis in epithelial ovarian cancer via the PI3K-AKT pathway. Eur Rev Med Pharmacol Sci. 2017;21(14): 3176-3184.

23. Wu DI, Wang T, Ren C, et al. Downregulation of BC200 in ovarian cancer contributes to cancer cell proliferation and chemoresistance to carboplatin. Oncol Lett. 2016;11(2):1189-1194.

24. Li J, Yang C, Li Y, Chen A, Li L, You Z. LncRNA GAS5 suppresses ovarian cancer by inducing inflammasome formation. Biosci Rep. 2018;38(2):BSR20171150.

25. Huang S, Qing C, Huang Z, Zhu Y. The long non-coding RNA CCAT2 is up-regulated in ovarian cancer and associated with poor prognosis. Diagn Pathol. 2016;11(1):49.

26. Tang W, Shen Z, Guo J, Sun S. Screening of long non-coding RNA and TUG1 inhibits proliferation with TGF- $\beta$ induction in patients with COPD. Int J Chron Obstruct Pulmon Dis. 2016;11:2951-2964.
27. Slaby O, Laga R, Sedlacek O. Therapeutic targeting of non-coding RNAs in cancer. Biochem J. 2017;474(24):4219-4251.

28. Li J, Yu H, Xi M, Lu X. Long noncoding RNA C17orf91 is a potential prognostic marker and functions as an oncogene in ovarian cancer. J Ovarian Res. 2016;9(1):49.

29. Tani H, Torimura M. Identification of short-lived long non-coding RNAs as surrogate indicators for chemical stress response. Biochem Biophys Res Commun. 2013;439(4):547-551.

30. Tani H, Torimura M. Development of cytotoxicity-sensitive human cells using overexpression of long non-coding RNAs. J Biosci Bioeng. 2015;119(5):604-608.

31. Zhang JH, Wei HW, Yang HG. Long noncoding RNA SNHG15, a potential prognostic biomarker for hepatocellular carcinoma. Eur Rev Med Pharmacol Sci. 2016;20(9):1720-1724.

32. Ma Z, Huang H, Wang J, et al. Long non-coding RNA SNHG15 inhibits P15 and KLF2 expression to promote pancreatic cancer proliferation through EZH2-mediated H3K27me3. Oncotarget. 2017;8(48): 84153-84167.

33. Tani H, Okuda S, Nakamura K, Aoki M, Umemura T. Short-lived long non-coding RNAs as surrogate indicators for chemical exposure and LINC00152 and MALAT1 modulate their neighboring genes. PLoS One. 2017;12(7):e0181628.
OncoTargets and Therapy

\section{Publish your work in this journal}

OncoTargets and Therapy is an international, peer-reviewed, open access journal focusing on the pathological basis of all cancers, potential targets for therapy and treatment protocols employed to improve the management of cancer patients. The journal also focuses on the impact of management programs and new therapeutic agents and protocols on

\section{Dovepress}

patient perspectives such as quality of life, adherence and satisfaction. The manuscript management system is completely online and includes a very quick and fair peer-review system, which is all easy to use. Visit http://www.dovepress.com/testimonials.php to read real quotes from published authors. 